Cross Sections and Analyzing Powers in Deuteron Elastic Scattering 
This report was prepared as an account of work sponsored by the United States Government. Neither the United States nor the United States Atomic Energy Commission, nor any of their employees, nor any of their contractors, subcontractors, or their employees, makes any warranty, express or implied, or assumes any legal liability or rusponsibility for the accuracy, completeness or usefulness of any information, apparatus, product or process disclosed, or represents that its use would not infrings privately owned rights.

Printed in the United States of America. Available from

National Technical Information Service

U. S. Department of Commerce

5285 Port Royal Road

Springfield, Virginia 22151

Price: Printed Copy \$3.00; Microfiche \$0.95 
LA-5051

UC.34

ISSUED: October 1972

NOTICE

This repurt was prepared as an account of work sponsored by the United States Government. Neithe: the United States nor the United States Atomic Enersy Commission, nor any of their employees, nor any of their contractors, subcontractors, or their employees, makes any warranty, express or implied, or assumes any legal liability or rosponsibility for the accuracy, completenoss or usefulneas of any tinformation, pleteness or usefulness of any thromatlon, apparatus, product or procoss dicelses, or ropresents that lis use would not infrings privately owned rights.

\section{Cross Sections and Analyzing Powers in Deuteron Elastic Scattering}

by

R. A. Hardekopf

D. D. Armstrong

L. L. Catlin

P. W. Keaton, Jr.

G. P. Lawrence 
CROSS SECTIONS AND ALALYZING POWERS IN DEUTERON ELASTIC SCATTERING

by

R. A. Hardekopf, D. D. Armstrong, L. L. Catlin,

P. W. Keaton, Jr., and G. P. Lawrence

ABSTRACT

All tensor analyzing powers have been measured for $15-\mathrm{MeV}$ deuterons elastically scattered from ${ }^{4} \mathrm{He},{ }^{52} \mathrm{Cr},{ }^{56} \mathrm{Fe},{ }^{60} \mathrm{Ni},{ }^{90} \mathrm{Zr}$, ${ }^{122} \mathrm{Sn}$, and ${ }^{197} \mathrm{Au}$. The results are presented in tabular and graphical form along with relative cross sections obtained for all but ${ }^{4} \mathrm{He}$.

\section{INTRODUCTION}

The data presented heic were obtained as part of an iuvestigation of the deuteron optical-model potential.1,2 A complete and consistent set of leuteron-scattering data on several nuclei at one energy were required for comparison with opticalmodel calculations. ${ }^{3}$ Although an optical-model fit to the deuteron differential cross section and analyzing tensors could be credible only if it spanned several energies as well as several atomic numbers, to date no one has obtained a fit to these five observables at a single energy for any targat nucleus.

The data acquisition was made possible by the development at the Los Alamos Scientific Laboratory (LASL) of a high-intensity, Lamb-shift polarized ion source, ${ }^{4}$ a rotating scattering chamber, and rapid methods for reliable determination of all four analyzing powers in deuteron-induced reactions. ${ }^{3,5}$

Except for helium, all targets were isotopically enricied metallic foils $\sim 0.1$ to $0.5 \mathrm{mg} / \mathrm{cm}^{2}$ thick. The helium was contained at 1.0 atm in a gas-scattering cell made of $0.0025-m m-t h i c k$ HAVAR* foil.

*HAVAR is the trade name of $\varepsilon$ high-tensile-strength alloy manufactured by the Hamilton Watch Co.
Four detector telescopes at azimuthal positions corresponding to left, right, up, and down were used. The LASI tandem on-line computer controlled the reaction-chamber rotation sequence, the deuteron-beam nuclear magnetic substate, $\mathrm{m}_{I}$, and measurement of the beam polarization. ${ }^{5}$ Beam polarizations ${ }^{6}$ of typically $80 \% \mathrm{~m}_{\mathrm{I}}=+1$ or 0 , were used for the measurements. The notation and coordinate systems used are consistent with the Madison Convention.?

The data are presented both in tabular form and graphically. We assign an absolute uncertainty of \pm 0.02 to all of the analyzing-power data. Statistical uncertainties for these data were generally less than \pm 0.01 . Only relative differential cross sections were measured, and these data are assigned a relative uncertainty of $5 \%$. This estimate includes contributions from counting statistics as well as beam integratior. and dead-time correction. The cross-section anguJar distributions have been normalized to opticalmodel predictions. ${ }^{3}$

\section{PISTRENCES}

I. P. W. Keaton, Jr., E. Aufdembrink, and L. R. Veeser, Los Alamos Scientific Laboratory report LA-4379-MS (1970).

2. P. W. Keaton, Jr., and D. D. Armstrong, to be published. 
3. D. D. Armstrong, R. A. Hardekopf, and P. W. Keaton, Bull. Am. Phys, Soc, 11, 686 (1972); and to be published.

4. J. L. McKibben, G. P. Lawrence, and G. G. Ohisen, Third Sygpasium Polarization Phenomena In Nuclear Reactions (Univ. of Wisc. ?ress, Madison, 1971) p. 828; G. P. Lawrence, G. G. Oblsea, and J. L. McKibben, Phys. Letters 28B, $594(1969)$.

5. G. P. Lawrence, G. G. Ohlsen, J. L. McKibben, P. W. Keaton, Jr., and D. D. Armstrong, Third
Symostum Polarization Phenomens in Nuclear Resctions (Univ, of W1:BC. Press, Madison, 1971) p. 855 .

6. G. O. Ohlsen, G. P. Lakrence, P. W. Keaton, Jr., J. L. McKJbben, and D. D. Armstrong, ibid., p. 842 .

7. Madison Convention, ibial.

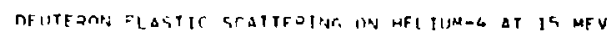

\begin{tabular}{|c|c|c|c|c|}
\hline THETAITNI & sir) & $\Delta(2)\}$ & $A(x)$ & $.54(x x-y y)$ \\
\hline $20.9 !$ & -.014 & -.008 & . nas & -.080 \\
\hline$M .72$ & -.054 & $-.0 x A$ & .070 & -.178 \\
\hline 44.57 &.$->27$ & $-.10 n$ & .048 & $-.74 n$ \\
\hline $60.1 \mathrm{~N}$ & $-.4>4$ & -.197 & $.00 n$ & -.>ด? \\
\hline $51.7 R$ &.$- A R Q$ & -.110 & -.111 & -.757 \\
\hline $\begin{array}{l}55.717 \\
5 \% .87\end{array}$ & -.144 & -.404 & -.171 & $=.257$ \\
\hline $\begin{array}{l}5 A . A 7 \\
B>.79\end{array}$ & $\begin{array}{l}-.575 \\
-.114\end{array}$ & $\begin{array}{l}-.344 \\
-.131\end{array}$ & -.077 & -.000 \\
\hline $\begin{array}{l}B>79 \\
B 5.94\end{array}$ & $-.14 ?^{3}$ & $\begin{array}{r}-.131 \\
.014\end{array}$ & $\begin{array}{l}011 \\
.097\end{array}$ & .061 \\
\hline $\begin{array}{l}B 5,94 \\
7>, A ?\end{array}$ & .051 & jo? & .0A7 & .130 \\
\hline $\begin{array}{l}7>.47 \\
70.76\end{array}$ & noi & & .707 & .243 \\
\hline 70.16 & .004 & - Jan & .277 & .335 \\
\hline $\begin{array}{l}95.41 \\
97.17\end{array}$ &. $\ln ?$ &.$>0 n$ & . Pant & .411 \\
\hline 97.17 &.$- .0 \mathrm{OH}$ & $.16 \mathrm{H}$ & .257 & .450 \\
\hline 90.77 &.$- n$ chn &.$>03$ & .751 & .510 \\
\hline $104.0 R$ & -.104 & $.24 A$ & .731 & . 570 \\
\hline 100.71 &.$->4 \%$ & .251 & 1 & . 570 \\
\hline 115.08 & -.374 & .234 & .149 & . $\triangle A O$ \\
\hline $12 n .21$ & -.701 & .240 & .147 & .416 \\
\hline $125.0^{\circ}$ & -.947 & .277 & $.17 R$ & $.2 \times 1$ \\
\hline $1 ? 0.71$ & -.177 & .214 & . & . Ono \\
\hline 1314.04 & .DPh & . IAA & (1) 84 & -.177 \\
\hline 170.73 & .175 & . Ia & .077 & -.737 \\
\hline 142.17 & $.57 A$ & $.11 ?$ & .050 & -.467 \\
\hline 145.87 & .AR5 & .100 & .044 & $-.45 n$ \\
\hline 140.76 & .402 & .117 & .116 & -.397 \\
\hline 157.67 & - ASG & .124 & GAP & -.317 \\
\hline 155.94 & - nor & .071 & .000 & -.757 \\
\hline 141.78 & .477 & .070 & .074 & -.124 \\
\hline $167.7 A$ & .114 & .093 & .067 & -.072 \\
\hline
\end{tabular}

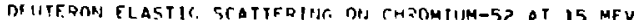

\begin{tabular}{|c|c|c|c|c|c|}
\hline THF TA(CN) & $\mid 4 m / 50)$ & $A(x)$ & $A(2)$ & $A(x)$ & $.5 \Delta(x x-y y)$ \\
\hline $\begin{array}{l}19.70 \\
17.45 \\
15.59 \\
70.74 \\
75.94 \\
11.11 \\
76.71\end{array}$ & 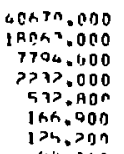 & & & & \\
\hline 41.67 & GA. $9 \mathrm{An}$ & .027 & -.096 & $.00 x$ & -.017 \\
\hline 46.57 & 57.500 & -.001 & -.004 & -.007 & -.014 \\
\hline $\begin{array}{l}51.70 \\
54,92\end{array}$ & $\begin{array}{l}27.710 \\
14.450\end{array}$ & $\begin{array}{l}\because 074 \\
\therefore 194\end{array}$ & .014 & .044 & $=080$ \\
\hline $\begin{array}{l}4 h .9 ? \\
01.0 ?\end{array}$ & $\begin{array}{r}14.450 \\
4 . m>1\end{array}$ & $\begin{array}{l}\because 104 \\
-.72 n\end{array}$ & $\begin{array}{l}.072 \\
.001\end{array}$ & $\begin{array}{l}.005 \\
.087\end{array}$ & $\begin{array}{l}=044 \\
=028\end{array}$ \\
\hline 17.01 & S.AR? & $-.04 \mathrm{~h}$ & -.014 & .030 & $\begin{array}{l}-.097 \\
=.09 ?\end{array}$ \\
\hline 72.00 & 3.79 & .744 & . ПRम & .007 & $\because 0 A B$ \\
\hline 77.15 & $\begin{array}{l}3.484 \\
? .918\end{array}$ & .209 & .040 & $=.006$ & -.074 \\
\hline 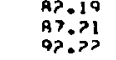 & $\begin{array}{l}? .918 \\
3.005 \\
? .600\end{array}$ & $\begin{array}{r}.067 \\
\because .191 \\
-.74 x\end{array}$ & $\begin{array}{r}.077 \\
-.079 \\
-.173\end{array}$ & $\begin{array}{l}=.054 \\
-.005 \\
.004\end{array}$ & $\begin{array}{r}.015 \\
\because .073 \\
-.107\end{array}$ \\
\hline 97.71 & $1.4 k 1$ & -.405 & -.111 & .049 & $\begin{array}{l}-.107 \\
-.107\end{array}$ \\
\hline 107.19 & .607 & -.778 & $-.27 \mathrm{~A}$ & .177 &.$->91$ \\
\hline 107.15 & .773 & $.7 A 3$ & $.0+n$ & -.007 & -.105 \\
\hline 112.00 & .517 & .496 & .177 & -.074 & $-.07 ?$ \\
\hline 137.01 & .757 & $.17 h$ & .174 & -.055 & .970 \\
\hline 121.97 & .950 & $-.09 A$ & -.001 & -.076 & -.037 \\
\hline IPA.AP & . $A 1 A$ & -.713 & -.095 & -.047 & -.057 \\
\hline 171.70 & $\begin{array}{l}555 \\
.75 n\end{array}$ & -.571 &.$->77$ & .058 & $=.125$ \\
\hline 141.47 & ה & $\begin{array}{l}\because .540 \\
\because .50 ?\end{array}$ & $\begin{array}{l}\because .771 \\
-.345\end{array}$ & $\begin{array}{l}-.067 \\
-.106\end{array}$ & $\begin{array}{l}-.74 R \\
-.774\end{array}$ \\
\hline $14 k .77$ & $.14 \mathrm{~A}$ & .107 & .072 & .075 & .017 \\
\hline 151.11 & 100 & .401 & .417 & .207 & .309 \\
\hline & פ & .789 & .296 & .146 & .277 \\
\hline
\end{tabular}

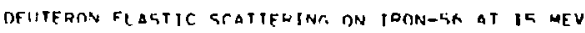

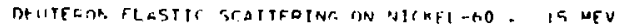

\begin{tabular}{|c|c|c|c|c|c|}
\hline IHF TA $(T, N)$ & $(40,50)$ & $A(r)$ & $\Delta(2)$ & $A(\times 2)$ & $.54(x x-r y)$ \\
\hline $\begin{array}{l}10.76 \\
17.47 \\
15.57 \\
20.71 \\
25.87 \\
71.07 \\
76.18\end{array}$ & 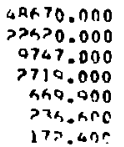 & & & & \\
\hline $\begin{array}{l}76.19 \\
61.73\end{array}$ & $\begin{array}{l}177.4 n e \\
1>31.90\end{array}$ & -.007 & - D? & .007 & $-.0>5$ \\
\hline $\begin{array}{l}49.44 \\
51.52\end{array}$ & $\begin{array}{l}n+7>0 \\
\because>>0\end{array}$ & $\begin{array}{l}\because r 11 \\
.0 \times a\end{array}$ & $\begin{array}{r}-.006 \\
.0>0\end{array}$ & $\begin{array}{l}.010 \\
.041\end{array}$ & $\begin{array}{l}\because 040 \\
-040\end{array}$ \\
\hline $\begin{array}{l}56 . \times 0 \\
51.73\end{array}$ & 16.4000 & $\begin{array}{l}-.174 \\
-.077\end{array}$ & $\begin{array}{l}.074 \\
.077\end{array}$ & $\begin{array}{l}.075 \\
.063\end{array}$ & $\begin{array}{l}-.048 \\
-.048\end{array}$ \\
\hline $6 x .97$ & $\begin{array}{r}16.450 \\
7.454\end{array}$ & $\begin{array}{l}-.077 \\
.074\end{array}$ & $\begin{array}{l}.077 \\
.007\end{array}$ & $\begin{array}{l}.063 \\
.061\end{array}$ & $\begin{array}{l}-.048 \\
-.038\end{array}$ \\
\hline 71.94 & $9.77 \mathrm{~K}$ & .100 & .015 & .010 & -.059 \\
\hline $\begin{array}{l}78.99 \\
\text { B?.0? }\end{array}$ & $\begin{array}{l}4.119 \\
7 . \operatorname{mat}\end{array}$ & -100 & $=.001$ & -.000 & -.015 \\
\hline A7.0x & $>.907$ & $\begin{array}{l}-.115 \\
-.264\end{array}$ & $\begin{array}{l}-.074 \\
-.087\end{array}$ & $\begin{array}{r}-.074 \\
.014\end{array}$ & $\begin{array}{r}.041 \\
-.056\end{array}$ \\
\hline $97.0 \mathrm{n}$ & 3.114 & -.779 & $-.1>5$ & קו & -.095 \\
\hline $\begin{array}{r}97.04 \\
107.07\end{array}$ & $\begin{array}{l}1.054 \\
.54 a\end{array}$ & $\begin{array}{r}-.746 \\
.705\end{array}$ & $\begin{array}{l}0.000 \\
0.000\end{array}$ & $\begin{array}{l}.070 \\
.0 .76\end{array}$ & $\begin{array}{l}\because .159 \\
-.036\end{array}$ \\
\hline $\begin{array}{l}106.99 \\
111.94\end{array}$ & $.91 \mathrm{~A}$ & .408 & 0.000 & $=05 A$ & .137 \\
\hline $\begin{array}{l}111.94 \\
119.87\end{array}$ & & $\begin{aligned} \because 179 \\
\therefore \text {. }\end{aligned}$ & $\begin{array}{l}0.000 \\
0.000\end{array}$ & $\begin{array}{l}-.041 \\
-.004\end{array}$ & $\begin{array}{r}.075 \\
\therefore .05 ?\end{array}$ \\
\hline 121.70 & . & -.746 & 6.000 & .000 & -.157 \\
\hline 12K.KS & & -.797 & 0.000 & .077 & -.194 \\
\hline 171.59 & .604 & -.475 & $0.0 \times 0$ & -.004 & $=.754$ \\
\hline $175.4 \mathrm{~A}$ & & -.273 & 0.000 & - n>0 &. .195 \\
\hline 141.77 & .171 & .157 & 0.900 &.$- n 01$ & $-.03 ?$ \\
\hline 165.12 & & $.77 \mathrm{~s}$ & 0.000 & .039 &.$>70$ \\
\hline $151.0^{7}$ & .744 & $1 \mathrm{hag}$ & $0 . n n o$ & .018 & .305 \\
\hline
\end{tabular}

\begin{tabular}{|c|c|c|c|c|c|}
\hline ThETAICN, & $(M G / S D)$ & $A(n)$ & $4(77)$ & $A(x)$ & - SA $(x x-y Y)$ \\
\hline 41.25 & 141.204 & $-.0>0$ & $-.01 \mathrm{~F}$ & $.00 n$ & -.072 \\
\hline $4=17$ & 70,640 & -.044 & -.007 & .017 & .006 \\
\hline 51.40 & 20,007 & -.015 & .056 & $.04 \%$ & -.047 \\
\hline 59.53 & $17.0=0$ & $-.05 ?$ & .011 & .057 & -.087 \\
\hline 61,49 & 14.777 & $.0>1$ & .010 & . DLA & .017 \\
\hline AR. Th & $\mid 1.091$ & $.0 \times 5$ & .014 & .014 & -.011 \\
\hline 71.67 & M.A7? & .On: & 0.000 & .007 & -.071 \\
\hline$P A .8 T$ & 3.104 &.$- n 47$ & -.019 & .010 & -.077 \\
\hline B1.ज? & 4.507 & -.237 & $\because .044$ & . ก७k & $\because 0$. OA \\
\hline 95.91 & 8.177 & -.101 & -.005 & .079 & $-.10 A$ \\
\hline 91.94 & $1.9>0$ &.$-|8|$ & $-.04 ?$ & .091 & $-.10 A$ \\
\hline 94.93 & 1.200 & .144 & - $(6+)$ & .054 & .070 \\
\hline $13: 91$ & 1.014 & .127 & -1t? & -.074 & .101 \\
\hline $\begin{array}{l}106 . A 7 \\
111 . A=\end{array}$ & $\begin{array}{l}1.16 \mathrm{~A} \\
1.70 \mathrm{~A}\end{array}$ & $\begin{array}{r}15 R \\
-.0 R 0\end{array}$ & $\begin{array}{l}11 \% \\
: 00 ?\end{array}$ & $\begin{array}{l}\because 014 \\
-0.04\end{array}$ & $\begin{array}{r}\triangle O A O \\
-O O A B\end{array}$ \\
\hline iis. $7 \mathrm{k}$ & $1.0 \mathrm{OB}$ &.$->7 ?$ & -.156 & $=000$ & -.0146 \\
\hline $131 . \mathrm{KA}$ & A15 & $-.39 A$ &.$->n i$ & .317 & -.714 \\
\hline 129.50 & .505 & -.407 & $-.7 A 1$ & .074 & -.370 \\
\hline 131.40 & $.31 \mathrm{~A}$ & -.027 & -.739 & .107 & $-.32 h$ \\
\hline $13 \mathrm{k} .77$ & $.3 \times 1$ & $.31 n$ & $.17 \mathrm{~K}$ & . nal & -.015 \\
\hline $\begin{array}{l}141.75 \\
144.11\end{array}$ & $\begin{array}{l}\text { TCa } \\
: 195\end{array}$ & .794 & .378 & $\begin{array}{r}-030 \\
-027\end{array}$ & .146 \\
\hline $\begin{array}{l}44.07 \\
150.97\end{array}$ & .477 & $\begin{array}{l}: 70 ? \\
-.074\end{array}$ & $\begin{array}{l}.700 \\
.000\end{array}$ & $\begin{array}{l}-.077 \\
\because .05 \mathrm{~A}\end{array}$ & .155 \\
\hline 155.A7 & .411 & -.710 & $.03 k$ & $\because 0$ A4 & Ina \\
\hline
\end{tabular}




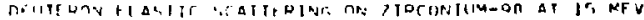

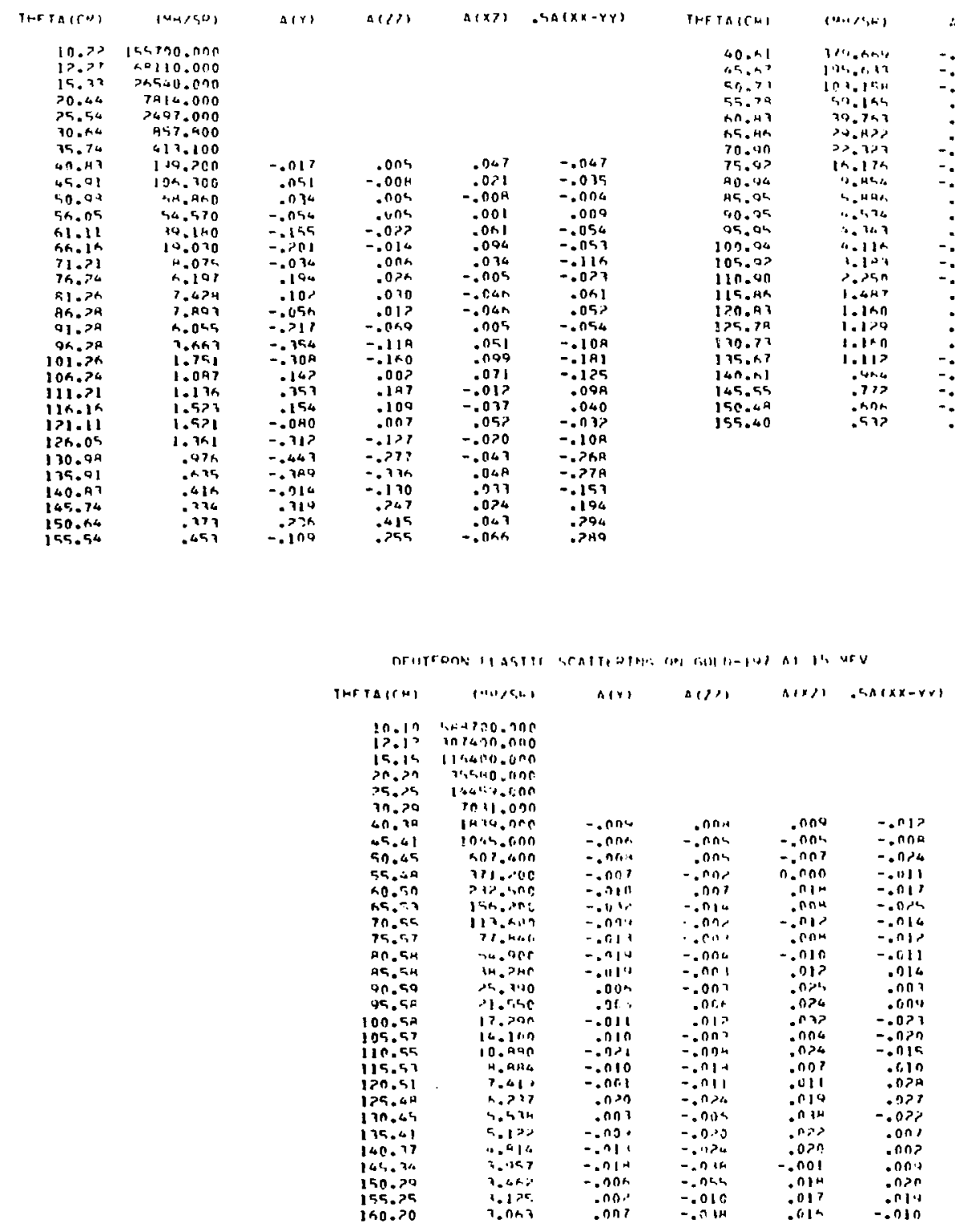



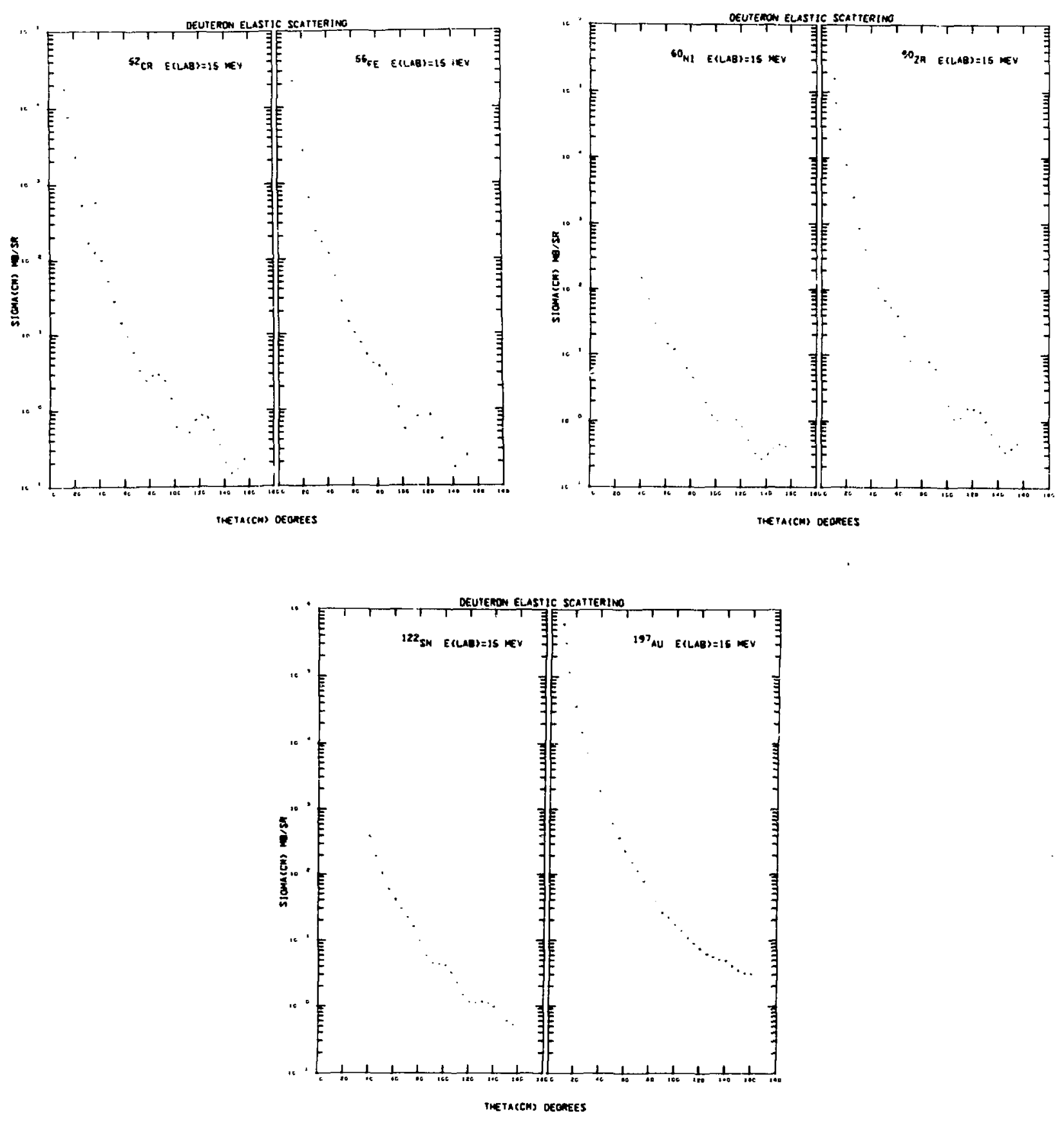


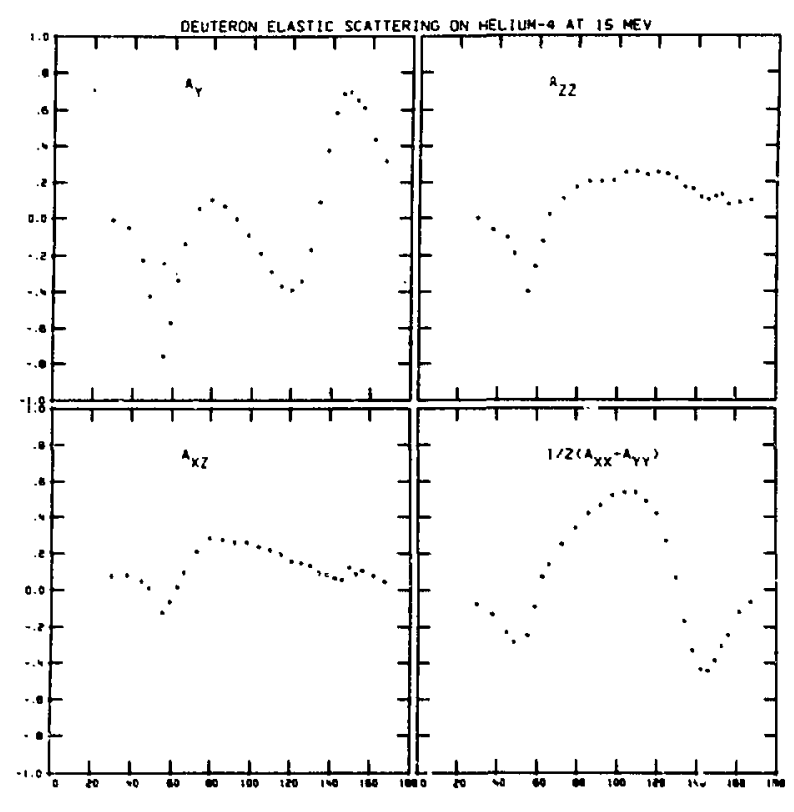

THETACLN) DEGREES

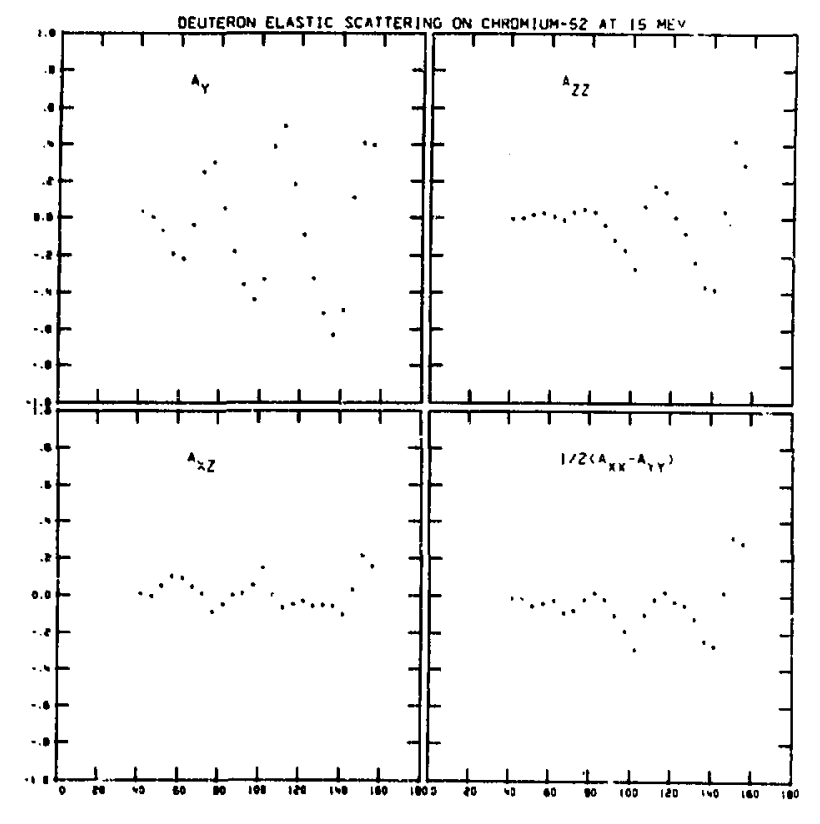

THETA(LM) DEGREES
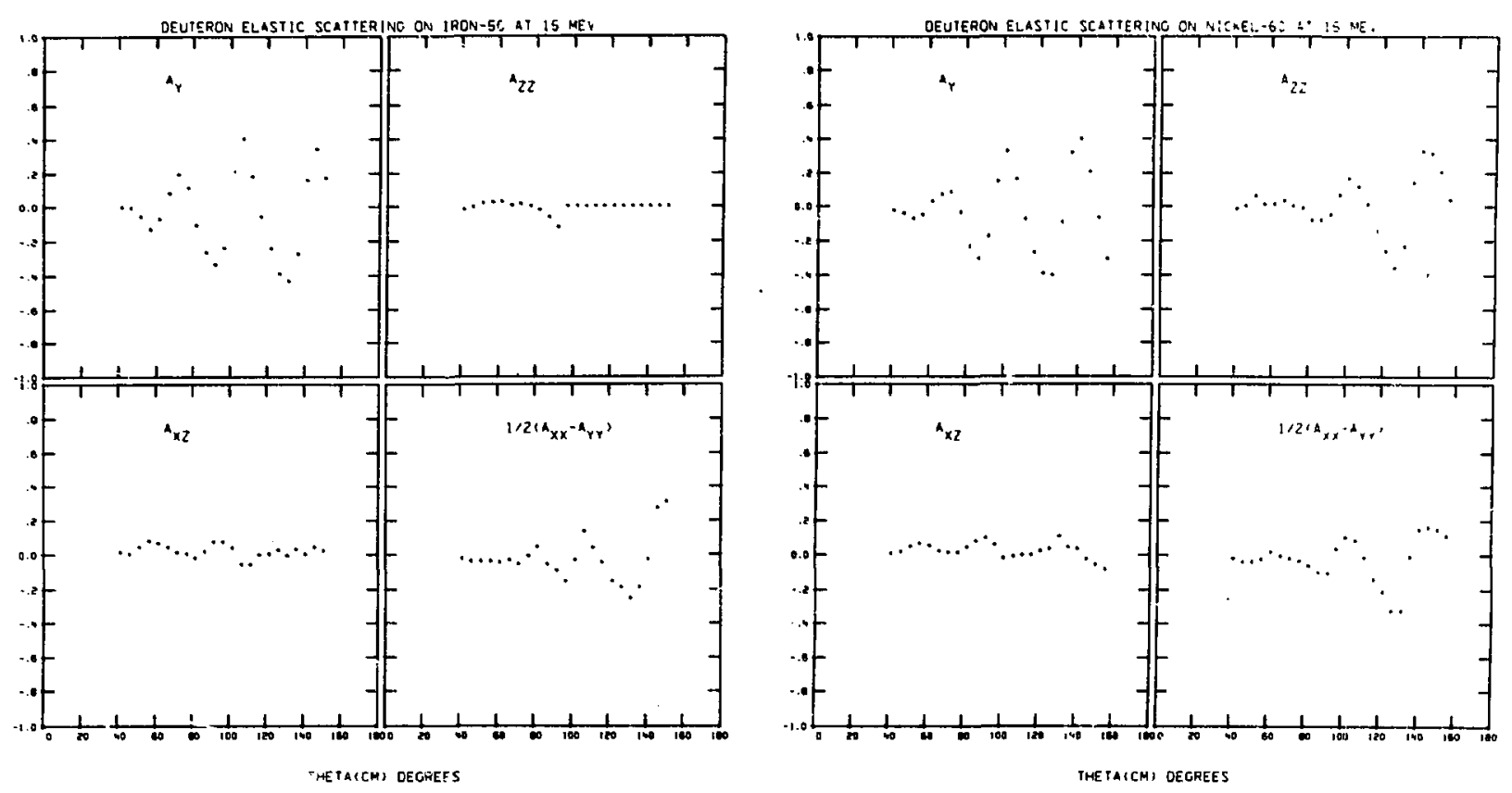


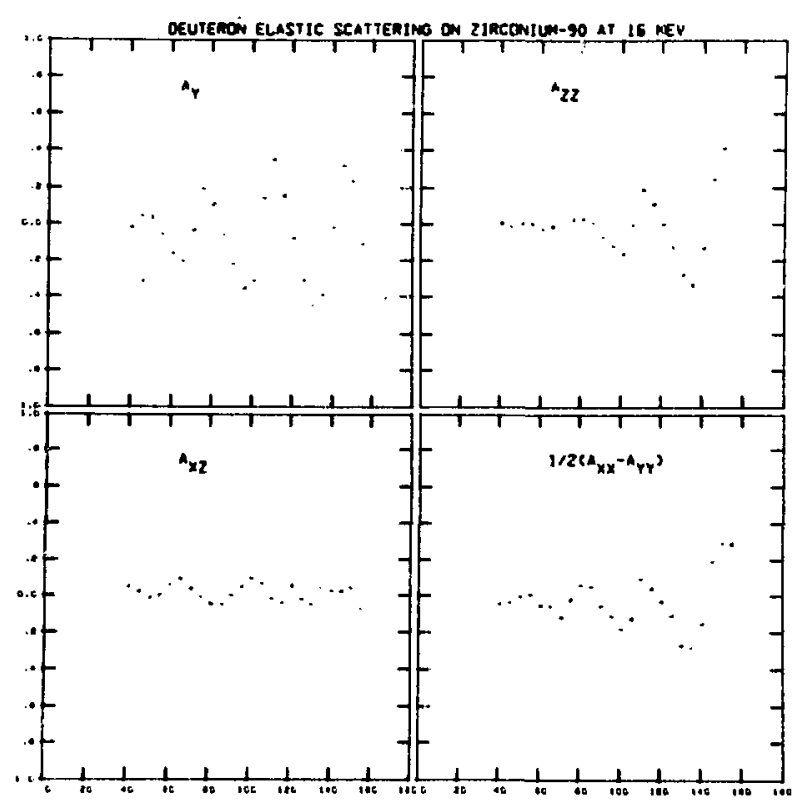

THE TACCM) DEOHEes

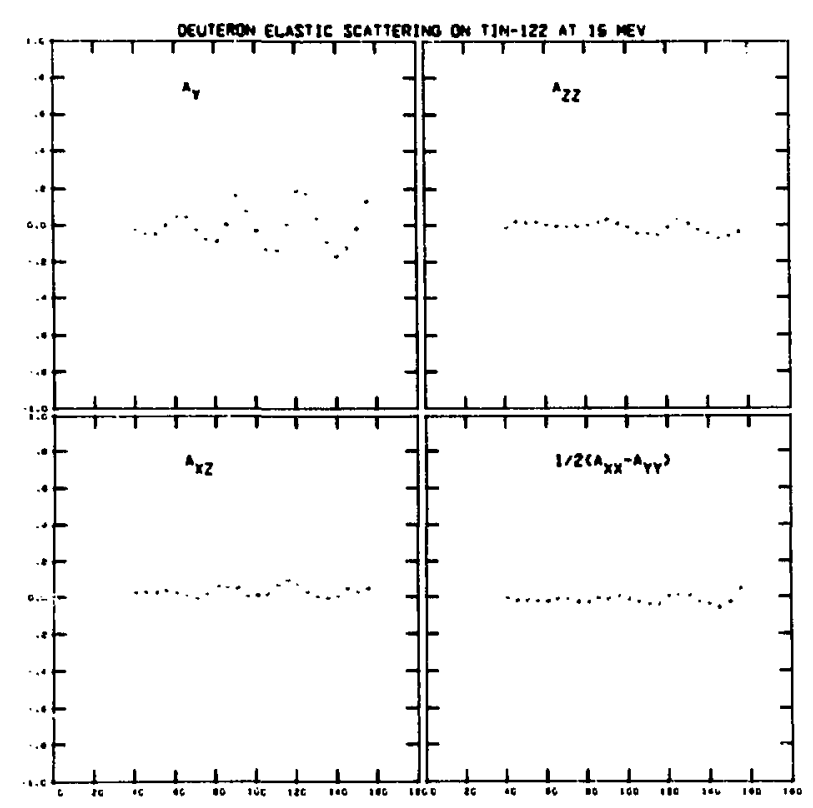

HETA(CH) OECMEES

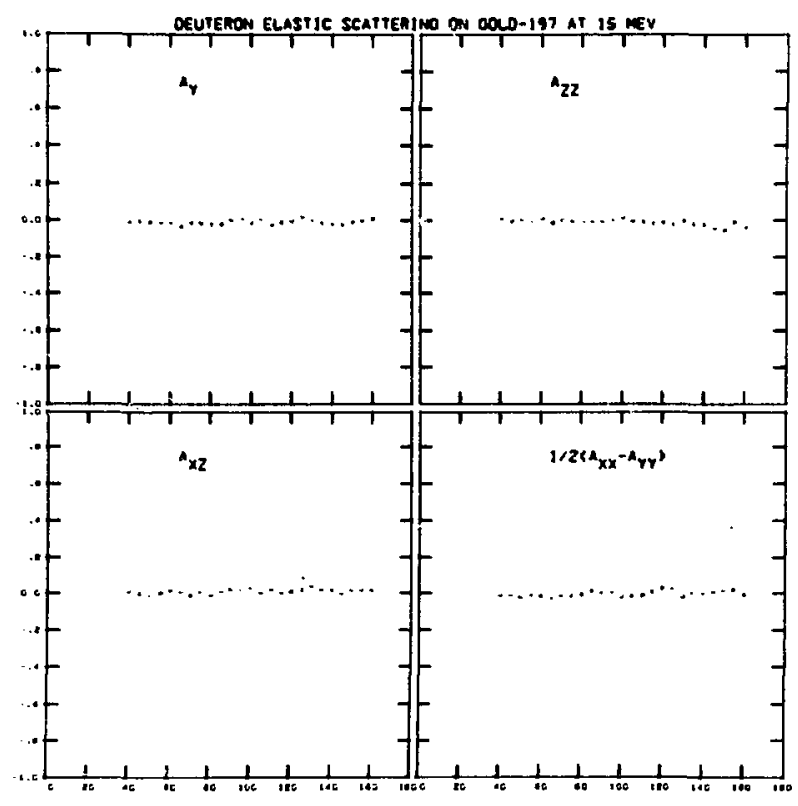

THETACEM OECREES 